East African Medical Journal Vol. 87 No. 3 March 2010

HOME-BASED HIV COUNSELLING AND TESTING IN WESTERN KENYA

S. Kimaiyo, MBChB, MMed, Moi University School of Medicine, Eldoret, Kenya, USAID-Academic Model Providing Access to Healthcare Partnership, Eldoret, Kenya, M.C. Were, MD, C. Shen, PhD, Indiana University School of Medicine, Indianapolis, USA, Regenstrief Institute, Inc., Indianapolis, Indiana, USA, S. Ndege, MD, MPH, USAID-Academic Model Providing Access to Healthcare Partnership, Eldoret, Kenya, Moi University School of Public Health, P.O. Box 4606-30100, Eldoret, Kenya, P. Braitstein, PhD, Moi University School of Medicine, Eldoret, Kenya, USAID-Academic Model Providing Access to Healthcare Partnership, Eldoret, Kenya, Indiana University School of Medicine, Indianapolis, USA, . Regenstrief Institute, Inc., Indianapolis, Indiana, USA, University of Toronto, Dalla Lana School of Public Health, Toronto, Canada, J. Sidle, MD, MS, J. Mamlin, MD, Moi University School of Medicine, Eldoret, Kenya, USAID-Academic Model Providing Access to Healthcare Partnership, Eldoret, Kenya, Indiana University School of Medicine, Indianapolis, USA

Request for reprints to: Dr. P. Braitstein, Moi University School of Medicine, P.O. Box 4606 - 30100, Eldoret, Kenya

\title{
HOME-BASED HIV COUNSELLING AND TESTING IN WESTERN KENYA
}

\author{
S. KIMAIYO, M. C. WERE, C. SHEN, S. NDEGE, P. BRAITSTEIN, J. SIDLE and J. MAMLIN
}

\begin{abstract}
Background: The World Health Organisation (WHO) estimates that only $12 \%$ of men and $10 \%$ of women in sub-Saharan Africa have been tested for HIV and know their test results. Home-based counselling and testing (HBCT) offers a novel approach to complement facility-based provider initiated testing and counselling (PITC) and voluntary counselling and testing (VCT) and could greatly increase HIV prevention opportunities. However, there is almost no evidence that large-scale, door-to-door testing is even feasible in settings with both limited resources and significant stigma around HIV and AIDS.

Objective: To describe our experience with the feasibility and acceptance of home-based HIV counselling and testing (HBCT) in two large, rural, administrative divisions of western Kenya.

Design: The USAID-AMPATH Partnership conducted population-based, house-to-house HIV counselling and testing in western Kenya between June 2007 and June 2009. All individuals aged $\geq 13$ years and all eligible children were offered HBCT. Children were eligible if they were above 13 years of age, and their mother was either HIVpositive or had unknown HIV serostatus, or if their mother was deceased or whose vital status was unknown.

Setting: Kosirai and Turbo Divisions, Rift Valley Province, Kenya.

Results: There were 47,066 households approached in 294 villages: $97 \%$ of households allowed entry. Of the 138,026 individuals captured, 101,167 individuals were eligible for testing: $89 \%$ of adults and $58 \%$ of children consented to HIV testing. The prevalence of HIV in these communities was 3.0\%:2.7\% in adults and 3.7\% among children. Prevalence was highest in the 36-45 year age group and was almost always higher among women and girls. All persons testing HIV-positive were referred to Academic Model Providing Access to Healthcare (AMPATH) for further assessment and care; all consenting persons were counselled on HIV risk-lowering behaviours.

Conclusion: Home-based HIV counselling and testing was feasible among this rural population in western Kenya, with a majority of the population accepting to get tested. These data suggest that scaling-up of HBCT is possible and may enable large numbers of individuals to know their HIV serostatus in sub-Saharan Africa. More research is needed to describe the cost-effectiveness and clinical impact of this approach.
\end{abstract}

\section{INTRODUCTION}

The World Health Organisation (WHO) estimates that only $12 \%$ of men and $10 \%$ of women in sub-
Saharan Africa have been tested for HIV and know their test results (1). Knowledge of HIV status can be important for several reasons. First, it can help facilitate access to support and care, improving 
the likelihood that HIV-positive patients receive antiretroviral treatment before they develop advanced immune suppression and that high risk children will be identified and referred (2). Second, HIV-positive pregnant women can beenrolled into programmes for prevention of mother-to-child transmission (PMTCT) of HIV, effectively reducing the number of newborns infected with the virus (3). Third, it may reduce the frequency of unprotected sexual intercourse and increase condom use by HIV-positive patients and discordant couples (4). Finally, knowledge of HIV prevalence can help in public policy planning and resource allocation.

For years, the primary mechanism for HIV testing in sub-Saharan Africa has been clientinitiated voluntary counselling and testing (VCT) (5). More recently, provider-initiated HIV testing and counselling (PITC) has been advocated in an effort to increase testing uptake (6). Unfortunately, both facility-based VCT and PITC have limited reach. These testing strategies depend on selfreferral into the healthcare system, and as a result many patients are identified when disease is advanced, thus reducing the probability of good clinical outcomes of antiretroviral treatment (7). In addition, numerous social, cultural, and psychological barriers - including stigma, lack of motivation, and concerns about confidentiality, limit access to VCT and PITC (8).

Home-based counselling and testing (HBCT) offers a novel approach to complement facilitybased PITC and VCT and could greatly increase HIV prevention opportunities (9-12). Hypothesized benefits of HBCT includeearlier diagnosis, easier identification of discordant couples, reduction of stigma as more people are tested, and cost-savings and convenience for individuals who only have to be at home to get tested. In recognition of this, national AIDS control programmes in developing countries are starting to recommend $\mathrm{HBCT}$ as a testing and counselling modality for HIV (13). However, there is almost no evidence that large-scale, door-to-door testing is even feasible in settings with both limited resources and significant stigma around HIV and AIDS.

The USAID-Academic Model Providing Access to Healthcare(AMPATH) Partnership initiated HBCT in two Divisions in Western Kenya. The primary goal was to increase uptake of HIV testing in the villages, so as to offer earlier HIV care and treatment to those infected and provide risk reduction counselling to all household residents. In this paper, we describe the results of this initial implementation phase of HBCT, and highlight generalizable lessons which can help in implementing large-scale, door-to-door HBCT in the developing world.

\section{MATERIALS AND METHODS}

Although this project was not initiated as a research study, we sought and received approval from the Institutional Review Boards of Indiana UniversityPurdue University at Indianapolis, USA, and the Institutional Research and Ethics Committee at Moi University School of Medicine, Eldoret, Kenya, for the purposes of communicating our findings.

Setting: AMPATH was initiated in 2001 as a joint partnership between Moi University School of Medicine (Eldoret, Kenya), the Indiana University School of Medicine (Indiana, United States), and the Moi Teaching and Referral Hospital (Eldoret, Kenya). TheUSAID-AMPATHPartnership wasinitiated in 2004 when AMPATH received ongoing funding through USAID and the United States President's Emergency Plan for AIDS Relief (PEPFAR). The initial goal of AMPATH was to establish an HIV care system to serve theneeds of both urban and rural patients and to assess the barriers to and outcomes of antiretroviral therapy. Details of the development of this programme have been described in detail elsewhere (14).

Theinitial areas chosen to pilot (Kosirai Division) and scale-up (Turbo Division) HBCT were based on a number of factors. The Kosirai Division is located in Nandi North District of Rift Valley Province and represents a rural setting in western Kenya (Figure 1). Kosirai Division is composed of nine locations, 24 sub-locations and 110 villages. This Division is home to the Mosoriot AMPATH clinic, the first rural clinic established by the USAID-AMPATH Partnership. AMPATH initiated HBCT in the Kosirai Division because it lies within a zone of low HIV prevalence compared to other USAID-AMPATH sites and therefore anticipated that existing clinic capacity could handle the increased demand for care and services resulting from increased identification of HIV-positive patients. In addition, there has been a strong relationship between healthcare providers in Mosoriot Health Centre and the Kosirai community. This enabled the required support of the community to conduct the pilot testing of such a large and innovative programme. Turbo Division in Rift Valley Province was chosen as the first roll out site because it forms the catchments for the AMPATH Turbo Clinic and because it is in a low HIV prevalence area. Turbo consists of seven locations, nine sub-locations, and 135 villages. Both Turbo and Mosoriot clinics are within a 45 minute drive from Moi Teaching and Referral Hospital (Kenya's second teaching and referral hospital) inEldoret, allowing ease of transport, communication, and referrals between centres. 
Figure 1

Map of USAID-AMPATH clinics, including Mosoriot (Kosirai) and Turbo clinics

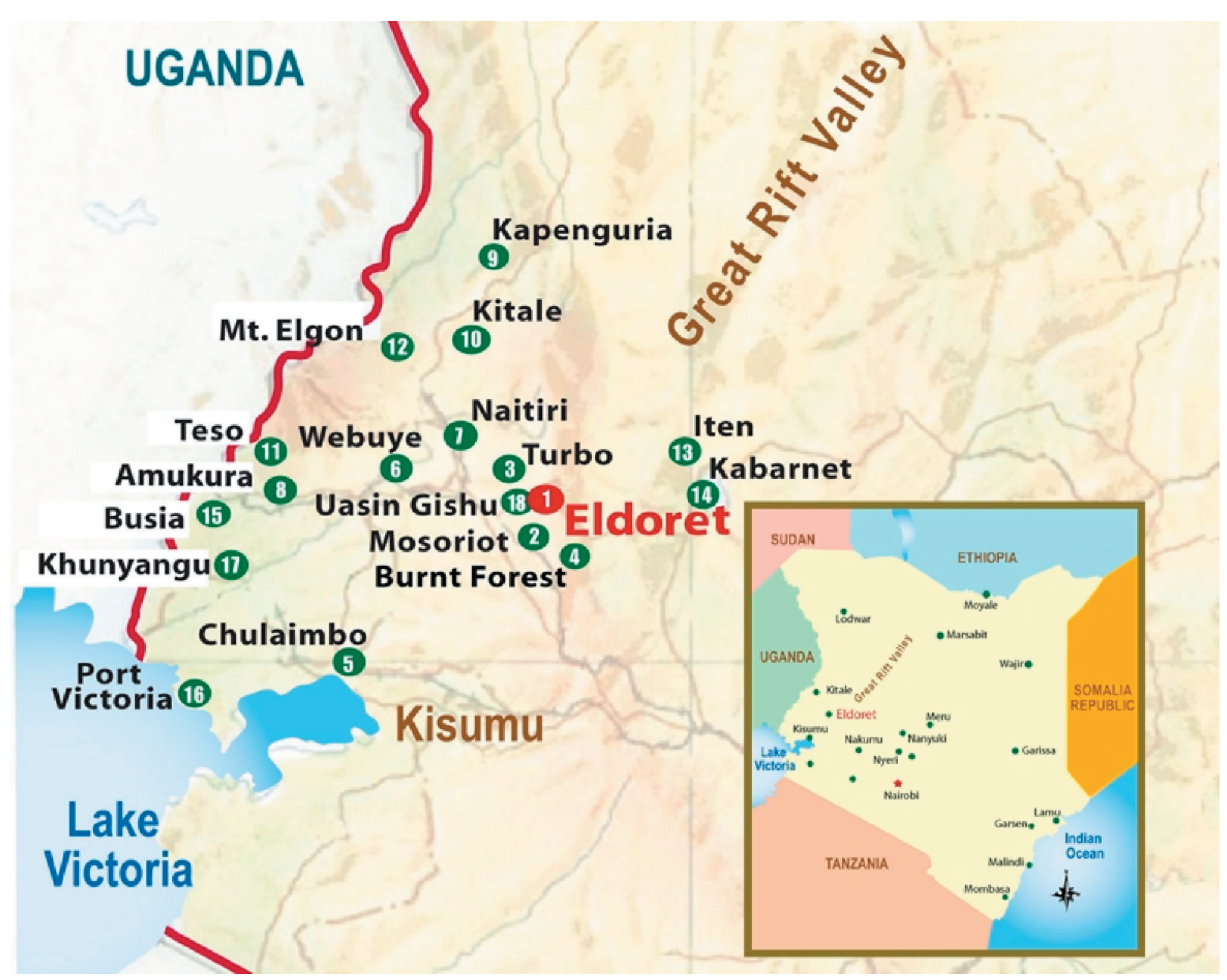

Turbo HBCT was conducted in two phases: JuneDecember 2008, and January-June, 2009. This was necessitated by the fact that time pressure initially led to many households not being surveyed, and many individuals in those households were missed since they were not found at home during the first counsellor visit. Post-hocit was agreed that counsellors would return to Turbo and offer counselling and testing to individuals and households that had been missed during the first phase and opt-in repeat testing for those previously tested.

Community mobilisation: It was presumed at the outset that population-wide HBCT would require a strong partnership between the institution performing $\mathrm{HBCT}$ and the target community. The first step in the HBCT process was therefore to gain insight into the community's perception of HBCT. This was done by conducting focus group discussions (FGDs) with community members using a pre-tested interview guide. Key leaders from the community (including chiefs, assistant chiefs, village elders, faith-based leaders, school heads, and other opinion leaders) were sensitised on the initiative during three additional sessions. The provincial administration (District officers, chiefs and assistant chiefs, village elders) together with other local community leaders all played critical roles in preparing the community for the visits. Major campaigns to mark the launch of HBCT were organised to create awareness and encourage community participation. In each of the villages, community mobilisation teams were formed and were made up of respected members of the community, included either a village elder, an adult male, an adult female, or a youth representative. This team visited every household in their respective villages and provided specific information about the HBCT programme. Specifically, they were able to connect the counsellors with the head of household, and requested permission for counsellors to visit the household for counselling and testing purposes.

Training of community mobilisers and counsellors: Given their role of disseminating accurate information about HIV/AIDS, AMPATH's services and the HBCT programme, the community mobilisers were 
trained to equip them with knowledge and skills in these areas to enable them carry out their functions. To conduct HBCT, 120 VCT Counsellors in Kosirai, and 90 in Turbo, were recruited, all with certification from the National AIDS and STD Control Program (NASCOP) in HIV counselling and testing and a minimum of one year field experience. The counsellors underwent formal training, through didactic and practical sessions in counselling skills as well as in the use of Personal Digital Assistant (PDA) devices and Global Positioning System (GPS) hardware used for data collection.

Door-to-door counselling and testing: Guided by a mobiliser, counsellors went to all households that had agreed to a visit within days of the mobilisation visit. Eligible individuals for counselling and testing were household residents 13 years of age and older. Children younger than 13 years whose mother was HIV-positive or whose HIV serostatus could not be ascertained, or whose mother was deceased or whose vital status was unknown were also regarded as eligible. Children younger than 18 months of age and eligible for testing were referred to the AMPATH clinic forDNAPCR testing after counselling of parents or guardians. A household resident was defined as an individual who spent at least one night in the household during previous period of one month.

Counsellors individually educated all eligible individuals (or parents/guardians for children) about HBCT and if they consented were given formal pre-test counselling. Clear and voluntary individual verbal consent for testing was also sought and documented for each individual. Consent for children 18 years of age or younger was obtained from their parents or guardians. In spite of the parents / guardian giving consent, children aged seven years and above also had to assent to the test. All adults were eligible for testing regardless of previous HIV test results unless there was documented evidence of a positive result. Pre- and post-test counselling was given to all tested individuals in situ as per standard national guidelines. Post-test counselling for those who are infected captured issues of preventing reinfection in themselves and preventing primary infection in others. For those testing HIV-negative, post-test counselling included behaviour change and the "ABC's" of HIV prevention. All persons testing HIV-positive were referred to AMPATH for care. Both HIV-positive and HIV-negative persons were counselled on risk-lowering behaviours.

The HIV testing algorithm for all eligible individuals entailed a parallel test using both the Determine HIV-1/2 rapid assay (Abbott Laboratories $\AA$ ) and SD Bioline HIV-1/23.0 (Standard Diagnostics Inc. ${ }^{\circledR}$, Kyonggi-do, South Korea) rapid assay. If the results were discordant, a tie-breaking test was done using the UniGold HIV test (Trinity Biotech ${ }^{\circledR}$, Dublin, Ireland) rapid assay.
Data collection: Counsellors conducting HBCT carried Palm T I X PDA devices (Palm Inc $®$, California, USA) which were used to enter standardised information into data-collection forms programmed with Pendragon Forms Software (DDH Software, Inc ${ }$, Florida, USA). Each household and individual, whether they consented to HIV counselling or not, received a unique identifier. Data collected included individual and household information, including GPS coordinates of the household via direct cable link to an external e-Trex GPS device (Garmin ${ }$, Kansas, USA). Each counselling and testing encounter took approximately 20 to 30 minutes depending on the clients level of understanding of HIV issues. At the end of each week, data was transferred from the PDA's to a Microsoft Access database (Microsoft Corp ${ }^{\circledR}$, Redman, Washington) by synchronizing the PDAs to a dedicated server behind a secure firewall.

Data analysis: Continuous variables were summarised by mean and standard deviation, while categorical variables were summarised by frequency and percentage. The primary outcomes of interest were eligibility for HIV testing, acceptance and uptake of HIV counselling and testing and HIV sero-prevalence within these communities. The data were analysed using SAS® version 9.1 (SAS Institute, Cary, North Carolina) and Stata/SE Version 10 (College Station, Texas).

\section{RESULTS}

The door-to-door census went to a total of 47,066 households in 294 villages in these rural Kenyan Divisions. Overall, 97\% of households allowed entry into the home by counsellors (Table1). There were17,863 individuals identified as household residents in Kosirai. Due to the pilot nature of the Kosirai phase, children were poorly captured in this Division. In the first phase of Turbo, 57,466 (29\% aged below 13 years) household residents were identified and in the second phase there were 62,697 (41\% aged under 13 years). The number of household residents, and children in particular, increased across the three phases as training and experience of the counsellors improved over time.

As summarised in Table 1, over half of the population during each of the three phases of HBCT was female. The median age was generally higher in women, but overall relatively young. Importantly, the median age dropped significantly in both men and women from Turbo Phase 1 to Turbo Phase 2, suggesting that youth and younger people were better captured during the second phase. In both Kosirai and the first phase of Turbo, approximately $20 \%$ of individuals had ever previously tested for HIV. The impact of the first phase of Turbo on the community's HIV testing history is evident in Phase 2, where the proportion of the population who had previously had an HIV test increased significantly to $33 \%$. 
Table 1

Summary of USAID-AMPATH Partnership HBCT population characteristics

\begin{tabular}{|c|c|c|c|}
\hline Indicator & Kosirai & Turbo Phase 1 & Turbo Phase 2 \\
\hline Number of households & 8936 & 20,909 & 17,221 \\
\hline Percent allowed to enter & $99 \%$ & $97 \%$ & $95 \%$ \\
\hline Total population captured & 17,863 & 57,466 & 62,697 \\
\hline$\geq 13$ years & 17,656 & 41,074 & 36,889 \\
\hline$<13$ years & 207 & 16,392 & 25,808 \\
\hline \multicolumn{4}{|l|}{ Gender } \\
\hline Male & $8511(48 \%)$ & $25,934(45 \%)$ & $29,744(47 \%)$ \\
\hline Female & $9352(52 \%)$ & $31,532(54 \%)$ & $32,953(53 \%)$ \\
\hline \multicolumn{4}{|c|}{ Median age (interquartile range) (years) } \\
\hline Male & $27(20-40)$ & $21(9-35)$ & $16(7-31)$ \\
\hline Female & $28(21-40)$ & $24(13-37)$ & $19(8-32)$ \\
\hline \multicolumn{4}{|l|}{ Testing history } \\
\hline Ever previously tested & $4183(23 \%)$ & $11,111(20 \%)$ & $20,521(33 \%)$ \\
\hline Never previously tested & $13,674(77 \%)$ & $45,604(80 \%)$ & $42,175(67 \%)$ \\
\hline
\end{tabular}

HIV testing eligibility: As summarised in Table 2, of the 138,026 individuals found through the three phases of HBCT, 101,167 individuals were identified as eligible for HIV counselling and testing, including 95,619 (100\%) adults and $5548(13 \%)$ children.

There were 17,682 individuals in Kosirai eligible for testing (52\% female) as per HBCT protocol (Table 2). Of these, 26 were children. There were
4,183 persons who had previously been tested for HIV (22 children, $30 \%$ of adult females, and $16 \%$ of adult males). Among those previously tested, 152 $(3.6 \%)$ had positive results ( $14 \%$ of previously tested children, $4.3 \%$ of females, and $2.0 \%$ of males). There were $120(79 \%)$ individuals with previously positive tests who were already receiving HIV care.

Table 2

Uptake of HIV counselling and testing through AMPATH HBCT

\begin{tabular}{|c|c|c|c|c|}
\hline Indicator & Kosirai & Turbo Phase 1 & Turbo Phase $2^{*}$ & Total \\
\hline \multicolumn{5}{|l|}{ Number of individuals eligible } \\
\hline for HIV C\&T as per HBCT protocol & 17,682 & 43,706 & 39,779 & 101,167 \\
\hline Adults & $17,656(100 \%)$ & $41,074(100 \%)$ & $36,889(100 \%)$ & $95,619(100 \%)$ \\
\hline Children & $26(\$)$ & $2632(16 \%)$ & $2890(11 \%)$ & $5548(13 \%)$ \\
\hline \multicolumn{5}{|l|}{ Number of individuals who accepted } \\
\hline HIV counselling (of those eligible) & $17,780(\$)$ & $42,586(97 \%)$ & $37,333(94 \%)$ & $97,699(97 \%)$ \\
\hline Adults & 17,649 & $40,604(99 \%)$ & $34,899(95 \%)^{* * * *}$ & * 93,152(97\%) \\
\hline Children & $131(\$)$ & $1982(75 \%)$ & $2434(84 \%)$ & $4547(82 \%)$ \\
\hline \multicolumn{5}{|l|}{ Number of individuals who accepted } \\
\hline HIV testing (of those eligible) & $18,593(96 \%)$ & 40,564 & 30,905 & $90,062(89 \%)$ \\
\hline Adults & $16,990(96 \%)$ & $39,148(95 \%)$ & $29,212(79 \%)^{* * * *}$ & $85,350(89 \%)$ \\
\hline Children & $130(95 \%)$ & $1416(54 \%)$ & $1693(59 \%)$ & $3239(58 \%)$ \\
\hline \multicolumn{5}{|l|}{ HIV seropositivity and HIV } \\
\hline seroprevalence $^{* *}$ & $409(2.9 \%)$ & $1482(3.7 \%)$ & $697(2.3 \%)$ & $2588(3.0 \%)$ \\
\hline HIV+ adults total & $397(2.3 \%)$ & $1408(3.6 \%)$ & $656(2.2 \%)$ & $2461(2.7 \%)$ \\
\hline Adults newly identified HIV+ & 316 & 1117 & 434 & 1867 \\
\hline HIV+ children ${ }^{* * *}$ Total & $12(9.2 \%)$ & $74(5.2 \%)$ & $41(2.4 \%)$ & $127(3.7 \%)$ \\
\hline Children newly identified HIV+ & 10 & 61 & 33 & 104 \\
\hline
\end{tabular}


${ }^{*}$ Of those approached in Phase 2 of Turbo, there were 19,064 adults who were tested in 2008, presumably in Turbo Phase 1.

$₫$ Please see the discussion section of the manuscript regarding counselling and testing of children in Kosirai. Although HBCT protocol was that any child whose mother died, had vital status unknown, or whose mother was HIV-positive or HIV serostatus unknown, only 26 children were identified as eligible for testing. There were in addition 105 children who accepted counselling at the request of the parent / guardian and are included in the data from Kosirai. Of the 131 children who accepted HIV counselling 130 also accepted testing.

${ }^{* *}$ Denominator for HIV prevalence is individuals tested

***There were an additional 253 children in phase 2, and 105 children in phase 1, who were tested at the request of the parent or guardian although they did not meet eligibility criteria as per HBCT protocol. Among these, one was HIV-infected.

${ }^{* * * *}$ Among individuals who were not tested in Phase 1 of Turbo, $98 \%$ of adults accepted HIV counselling in Phase 2, and 93\% accepted HIV testing. In contrast, among individuals who were tested during Phase 1 of Turbo, 91\% accepted HIV counselling, but only 66\% accepted HIV testing in Phase 2.

In the first phase of Turbo, there were a total of 43,706 individuals identified as eligible for HIV counselling and testing, including 41,074 (100\%) adults (55\% female) and $2632(16 \%)$ children. There were 11,111 individuals who had ever previously tested for HIV during this phase, including 174 children, 33\% of adult women and $19 \%$ of adult men. There were 614 individuals already known to be HIV-positive including 53 children: 558 (91\%) of these were already enrolled in AMPATH or other HIV programmes.

In Phase 2 of Turbo, 62,697 individuals were approached of whom 36,889 (59\%) were adults. In total there were 39,779 individuals in the $2^{\text {nd }}$ phase of Turbo eligible for testing, including 36,889 (100\%) adults (55\% female) and $2890(11 \%)$ children. There were 20,521 individuals who had been previously tested for HIV (including 614 children) of whom 95\% had been tested in 2008. These are assumed to have been tested through the first phase of HBCT in Turbo. Among all those previously tested, 1062 were known to be positive (including 118 children); 869 $(82 \%)$ were already enrolled in AMPATH or another HIV programme.

Counselling and testing: As summarised in Table 2, of the 101,167 adults and children eligible for HIV testing across the three phases of HBCT, $96.5 \%$ agreed to HIV counselling ( $97 \%$ of adults, $82 \%$ of children).

In Kosirai, 99\% of adults accepted HIV counselling, and 96\% accepted HIV testing. Although only 26 children were defined as eligible in Kosirai, an additional 105 parents/guardians requested counselling for them. Of the 131 children counselled, 130 accepted HIV testing.

During the first phase of Turbo, $99 \%$ of adults accepted HIV counselling and 95\% accepted HIV testing. There were also 2632 children aged less than 13 years who needed HIV testing as per the protocol.
Of these, $75 \%$ of parents or guardians consented to their being counselled about HIV testing, while only $54 \%$ consented to the actual testing.

In Phase 2 of Turbo, $94 \%$ of individuals accepted counselling, including 95\% of adults and $84 \%$ of parents/guardians on behalf of the children in their care. Of note, $91 \%$ of those tested in 2008 vs. $98 \%$ of those not tested in 2008 accepted HIV counselling, suggesting a lower uptake of HIV counselling among those more recently tested. Indeed, only $79 \%$ of adults overall consented to being tested during this second phase, but when stratified by whether they had been tested in 2008 (presumably through HBCT), 93\% of those not tested in 2008 opted for testing, but only $66 \%$ of those who were tested in 2008 agreed to HIV testing (in spite of the recommendation provided toall individuals tested through $\mathrm{HBCT}$ is to re-test in three months). Only 59\% of parents / guardians of eligible children consented to testing of the children.

Similar proportions of women $(95 \%$ Phase 1, $80 \%$ Phase 2) and men (96\% Phase 1, 78\% Phase 2) accepted HIV testing in Turbo.

HIV test results: Table 3 summarises the age and sex of those who tested HIV-positive in all three HBCT initiatives. There were 409 individuals who tested HIV-positive in Kosirai, of whom 316 were not previously known to be HIV-infected, in spite of several years of intensive community engagement. Of those 13 years and older, $397(2.3 \%)$ were HIV-positive, including $257(2.9 \%)$ of women, and $140(1.7 \%)$ of men. There were 12 children in Kosirai found to be HIV-positive $(9.2 \%), 10(83 \%)$ of whom were not previously known to be HIV-infected. Prevalence of HIV in adults peaked between ages $36-45$ years at $5.5 \%$. The peak age-group was the same for both females and males, with $6.4 \%$ and $4.4 \%$ prevalence respectively. 
Table 3

Prevalence of HIV among 90,062 individuals counselled and tested through HBCT

\begin{tabular}{|c|c|c|c|c|c|c|c|c|c|c|c|c|}
\hline \multirow{3}{*}{$\begin{array}{l}\text { Age category } \\
\text { (years) }\end{array}$} & \multicolumn{4}{|c|}{$\begin{array}{c}\text { Kosirai } \\
\text { HIV-positive }\end{array}$} & \multicolumn{4}{|c|}{$\begin{array}{l}\text { Turbo Phase } 1 \\
\text { HIV-positive }\end{array}$} & \multicolumn{4}{|c|}{$\begin{array}{c}\text { Turbo Phase } 2 \\
\text { HIV-positive }\end{array}$} \\
\hline & \multicolumn{2}{|c|}{ Male } & \multicolumn{2}{|c|}{ Female } & \multicolumn{2}{|c|}{ Male } & \multicolumn{2}{|c|}{ Female } & \multicolumn{2}{|c|}{ Male } & \multicolumn{2}{|c|}{ Female } \\
\hline & No. & $(\%)$ & No. & $(\%)$ & No. & $(\%)$ & No. & $(\%)$ & No. & $(\%)$ & No. & $(\%)$ \\
\hline Below 13 & 4 & 6.9 & 8 & 11.1 & 43 & 5.0 & 32 & 3.9 & 24 & 2.5 & 17 & 2.0 \\
\hline $13-18$ & 2 & 0.1 & 5 & 0.3 & 8 & 0.3 & 10 & 0.3 & 5 & 0.2 & 12 & 0.4 \\
\hline $19-25$ & 12 & 0.5 & 38 & 1.7 & 21 & 0.5 & 151 & 2.7 & 13 & 0.5 & 89 & 2.2 \\
\hline $26-35$ & 46 & 2.4 & 99 & 4.3 & 124 & 3.2 & 398 & 7.1 & 56 & 1.9 & 174 & 4.3 \\
\hline $36-45$ & 47 & 4.4 & 79 & 6.4 & 147 & 6.2 & 292 & 8.7 & 57 & 3.3 & 122 & 5.5 \\
\hline $46-55$ & 20 & 3.0 & 30 & 3.9 & 63 & 3.8 & 121 & 5.7 & 25 & 2.3 & 57 & 4.3 \\
\hline $55 \&$ above & 13 & 1.8 & 6 & 0.7 & 31 & 1.5 & 42 & 2.0 & 18 & 1.4 & 28 & 2.3 \\
\hline Total & 144 & 1.8 & 265 & 3.0 & 437 & 2.4 & 1046 & 64.6 & 198 & 1.4 & 499 & 2.9 \\
\hline
\end{tabular}

In Turbo Phase 1, 1408 (3.6\%) of those aged 13 years and over were found to be HIV-infected, including $4.6 \%$ of women, and $2.4 \%$ of men. In addition, $74(5.2 \%)$ of children tested were found to be HIV-infected. In total there were 1178 newly identified people with HIV. Adult HIV prevalence again peaked in the $36-45$ year age group, with prevalence of $8.7 \%$ among women, and $6.2 \%$ among men.

In the second phase of Turbo, $656(2.4 \%)$ adults tested HIV-positive, including $2.9 \%$ of women and $1.4 \%$ of men. There were also $40(2.3 \%)$ children who tested HIV-positive. HIV prevalence again peaked in the 36-45 year age group, with prevalence of 5.5\% in women vs. $3.3 \%$ in men. In total, 467 people with HIV were newly identified.

Pregnancy: There were 1387 pregnant women identified in the first phase in Turbo, of whom 1318 $(95 \%)$ consented to HIV testing (95\%). Of these, 56 $(4.5 \%)$ were found to be positive; $45(80 \%)$ of these were newly identified. Of the 911 pregnant women identified in the second phase of Turbo, $738(81 \%)$ accepted HIV testing; 21 (4.5\%) were found to be positive (64\% newly identified). Only 53\% and 57\% of pregnant women identified had ever attended ANC in Turbo Phase 1 and 2 respectively (pregnant women were not specifically identified in Kosirai).

\section{DISCUSSION}

Our findings indicated that the implementation of house-to-house HIV counselling and testing is feasible in a rural sub-Saharan African setting. Of the 47,066 households approached, 97\% allowed entry into the home by counsellors. Of the 101,167 adults and children eligible for HIV testing, 97\% agreed to HIV counselling (97\% of adults, $82 \%$ of children), and $89 \%$ agreed to HIV testing (89\% of adults, and
58\% of children). Prevalence of HIV was $3.0 \%: 2.7 \%$ in adults and $3.7 \%$ in children. We identified a total of 2588 HIV-positive persons, of whom 1961 were previously unaware of their HIV-positive status. This includes 66 pregnant women not previously known to be HIV-infected who were promptly referred for follow-up HIV care and prevention of mother to child transmission (PMTCT) interventions.

These data support previous efforts at assessing the feasibility of HBCT and reinforce its ability to increase the uptake of HIV counselling and testing in a more private, non-biased, non-threatening environment to men, women, and children $(9,11$, $12,15)$. Our findings suggest that conducting HBCT as a widespread, community-based programme can be successful at identifying people living with HIV and referring them for care. Our findings have also identified several important trends that should be considered by HIV prevention and treatment programmes alike.

We observed that HIV prevalence in females aged 13 years and over was higher than that of males in every adult age category. This is consistent with previously reported gender ratios in national population-based HIV surveys in Kenya (16) and international estimates for sub-Saharan Africa in general. It is estimated that $61 \%$ of people living with HIV in sub-Saharan Africa are women (17). HIV risk reduction efforts aimed at young men and women need to be improved. With the trust of the community gained, and high risk individuals identified, our data demonstrate that HBCT presents a unique opportunity to intervene in high risk situations such as pregnancy, orphaned/vulnerable children, among others, thus greatly improving the possibility of favorable outcomes (18).

We targeted paediatric testing to those children at highest risk of HIV infection, meaning those who had an HIV-positive or deceased mother, 
or whose mother's vital and/or HIV status was unknown. Despite the high risk of HIV infection for this population, on average only $69 \%$ of parents or guardians consented to have the children tested. Our data show improvements in the uptake of counselling and testing over time as the counsellors became better trained and more experienced. Importantly, the prevalence of HIV went down among children tested during the $2^{\text {nd }}$ phase of Turbo, suggesting that higher risk children (their parents/guardians) self-selected to be tested during the $1^{\text {st }}$ phase. These findings point to the need to explore further parental attitudes toward paediatric HIV testing, and the best strategies to increase uptake of paediatric testing, particularly for high risk populations. The children counselled and tested in Kosirai should not be viewed as generalizeable: the very low numbers captured, documented as eligible, and offered counselling and testing are indicative of substantive problems in the data collection process.

In examining the USAID-AMPATH experience with $\mathrm{HBCT}$, we can identify several factors key to the success of population-wide HBCT. These include:

(i) strong collaboration with the community;

(ii) dedicated human-resource and financial commitment

(iii) concertedandon-going community mobilisation efforts;

(iv) appropriate training of providers (mobilisers, counsellors) at all levels

(v) well-thought out counselling and consenting protocols with special attention to human rights and ethical issues

(vi) appropriate referral mechanisms for all participants depending on results

(vii) well planned and executed electronic health records systems for data collection, research, and monitoring and evaluation. Stakeholders considering implementing HBCT in their communities should consider in advance the anticipated HIV prevalence in their community and the impact on healthcare facilities that the identification of large numbers of HIV-infected persons may have. We chose the locations for scaling-up in part based on the anticipated low prevalence: identifying HIV-infected persons in the community is one thing, and caring for them in the clinic is another.

While we were able to demonstrate the feasibility of implementing HBCT at the population level, the undertaking had many challenges. First, the speed with which a team moves through a household or community comes at a price: missed households, and missed individuals within a household. This forced us to return to Turbo Division, at extra financial cost, and ultimately at the price of more time. There were advantages to returning to Turbo for a $2^{\text {nd }}$ phase. We learned that it is crucial to return repeatedly to a given household to ensure that all eligible residents are offered the opportunity to get tested. Although the uptake of testing was lower among those who had been tested during the first round, a majority of individuals who had been previously tested did accept re-testing. The median age of the population dropped significantly between the $1^{\text {st }}$ and $2^{\text {nd }}$ phases of HBCT in Turbo, suggesting that more youth and young people were captured. We speculate that this may be due to reduced stigma associated with HIV testing during the $2^{\text {nd }}$ phase since so many of the community accepted HIV counselling and testing during the $1^{\text {st }}$ phase. This would suggest that programmes should consider conducting cycles of HBCT in the community in order to increase the uptake of HIV testing in sub-populations who may be more vulnerable to stigma, including youth. Other challenges which cannot be fully described here but which we were able to generally overcome include logistics, the stigma of HIV in the community, disclosure issues when a member of the household tests positive, financial costs, and linking patients to the care system when necessary.

There are limitations to the data that may affect theirinterpretation. First, the Kosirai experience was a pilot, and the data should not be taken to reflect a true HIV prevalence. We have no reliable census data for example, on the actual population of the division, and therefore do not have a reliable denominator. Second, the way the data were collected in phase 2 of Turbo makes it virtually impossible to identify the overlap in the populations between phase 1 and phase 2 , and the data from phase 2 should therefore be interpreted with caution. Third, although the quality of the counsellors skills presumably improved over time, we have no measure of the quality of the counselling and testing. It may therefore be that the poor rates of testing uptake among the children were due in some measure to the way the counsellors approached the family about testing the child. Detailed analyses of testing uptake inchildren, and by gender, are presently underway as separate manuscripts. Fourth, we do not have information about the socioeconomic status or educational background of individuals approached through $\mathrm{HBCT}$, nor dowe have reasons for households declining entry.

Insummary, wehave shown thatimplementation of HBCT in rural sub-Saharan Africa is a viable and effective way to identify HIV-infected persons not previously known to be positive, including pregnant women. Despite the challenges, the programme enjoyed a high rate of participation and had significant success in HIV case-finding, delivery of HIV prevention messages, and referrals for HIV care and treatment. HBCT offers an ideal opportunity to gather additional information and to provide care 
beyond HIV and AIDS. Future research is needed on the cost-effectiveness of this programme, with special attention paid to the financial implications of identifying persons earlier on in the course of their infection, preventing new HIV infections, and preventing all the downstream consequences of advanced HIV disease (economic disintegration, new orphans, higher HIV transmission due to higher viral loads, etc.). As community-based and facility-based primary care is extended throughout sub-Saharan Africa, programmes like HBCT can provide an excellent platform for informing the focus and priorities of that care, mechanisms for efficiently and effectively delivering that care, and enabling all citizens of countries like Kenya to realize their fundamental human right to health.

\section{ACKNOWLEDGEMENTS}

To the Kosirai, Turbo, and Mautuma communities, and community elders for supporting the HBCT initiative. Special thanks to the mobilisers and counsellors, and all staff involved with the HBCT project, especially P.Akhaabi, D.Amadi, R. Ayikukwei, F. Ayodi, L. Birgen, L. Boit, W. Bunde, Dr. H. Campbell, Dr. Jane Carter, Milly Chemelil, Viola Chepngeno, Nelly Cherobon, B. Cheruyoit, Dr. R. Einterz, Dr. J. Greene, J. Imamai, V. Jebet, Dr. A. Jepkemboi, F. Juma, I. Kalamai, E. Kemboi, J. Kibet, S. Kiberenge, M. Kibor, E. Kigotho, S. Kimwattan, R. Kioko, J. Koech, C. Magut, H. Matoke, J. Mureithi, M. Murto, P. Musili, M. Mutai, J. Njumwah, Dr. W. Nyandiko, C. Opiyo, A. Oseno, R. Ototo, D. Rono, H. Rono, J. Rugut, H. Shikuku, S. Simatwa, I. Tanui, Dr. W. Tierney, M. Wandabwa, J. Wachira, S. Wanyela and Z. Wephukhu.

TheHBCT programme was supported by grants from Abbott Laboratories, the Purpleville Foundation and the Global Business Coalition. USAID-PEPFAR supported care for those found to be HIV-positiveand Abbott Laboratories provided test kits and logistical support.

\section{REFERENCES}

1. World Health Organization, Towards Universal Access: Scaling Up Priority HIV / AIDS Interventions in the Health Sector. A Progress Report, W. Press, Editor. 2007, World Health Organization: Geneva.

2. De Zoysa, I., et al. Role of HIV counseling and testing in changing risk behavior in developing countries. Aids. 1995; 9 Suppl A: p. S95-101.

3. Chigwedere, P., et al., Efficacy of antiretroviral drugs in reducing mother-to-child transmission of HIV in
Africa: a meta-analysis of published clinical trials. AIDS Res. Hum. Retroviruses. 2008. 24: 827-837.

4. Weinhardt, L.S., et al. Effects of HIV counseling and testing on sexual risk behavior: a meta-analytic review of published research, 1985-1997. Am. J. Pub. Hlth. 1999; 89: 1397-1405.

5. Summers, T., et al. Voluntary counseling, testing, and referral for HIV: new technologies, research findings create dynamic opportunities. J. Acquir. Immune. Defic. Syndr. 2000; 25 Suppl 2: S128-S135.

6. World Health Organization and J.U.N.P.f.H.A. (UNAIDS), Guidance to Provider-Initiated HIV Testing and Counselling in Health Facilities. WHO, Editor. 2007: Geneva.

7. Braitstein, P., et al., Mortality of HIV-1-infected patients in the first year of antiretroviral therapy: comparison between low-income and high-income countries. Lancet. 2006. 367: p. 817-824.

8. Hamra, M., et al. The relationship between expressed HIV / AIDS-related stigma and beliefs and knowledge about care and support of people living with AIDS in families caring for HIV-infected children in Kenya. AIDS Care. 2005; 17: 911-922.

9. Bateganya, M.H., Abdulwadud, O.A. and Kiene, S.M. Home-based HIV voluntary counseling and testing in developing countries. Cochrane Database Syst. Rev. 2007; 4: p. CD006493.

10. Fylkesnes, K., et al. HIV counselling and testing: overemphasizing high acceptance rates a threat to confidentiality and the right not to know. AIDS. 1999; 13: 2469-2474.

11. Matovu, J.K., et al. The Rakai Project counselling programme experience. Trop. Med. Int. Hlth. 2002; 7: 1064-1067.

12. Wolff, B., et al. Evaluation of a home-based voluntary counselling and testing intervention in rural Uganda. Hlth Policy Plan. 2005. 20: 109-116.

13. Kenya., N.A.a.S.C.P.o. HIV Testing and Counselling Guidelines. 2008, Government of Kenya, Ministry of Health, Nairobi.

14. Einterz, R., et al. Responding to the HIV pandemic: The power of an academic medical partership. Acad. Med. 2007; 82: 812-818.

15. Were, W., et al. Home-based model for HIV voluntary counselling and testing. Lancet. 2003; 361: 1569.

16. Government of Kenya, C.B.o.S.C., Ministry of Health(MOH) [Kenya], and ORC Macro. 2004, Kenya Demographic Health Survey 2003. 2004, Government of Kenya, Central Bureu of Statistics (CBS), Ministry of Health (MOH), ORC Macro 2004: Calvarton, Maryland, Kenya.

17. World Health Organisation(WHO)/ Joint United Nations Programme for HIV / AIDS(UNAIDS), AIDS Epidemic Update. N. classification. Editor. 2007.

18. UNAIDS/WHO/UNICEF. Children and AIDS: Second stock taking report: Actions and Progress. 2008. 